\title{
IDENTIFICACIÓN, EVALUACIÓN Y PUESTA EN VALOR DE UN PATRIMONIO HIDRÁULICO SINGULAR: LAS GALERÍAS DRENANTES DE LA CUENCA DEL JÚCAR
}

\author{
Emilio Iranzo García \\ Miguel Antequera Fernández \\ Jorge Hermosilla Pla \\ ESTEPA, Departament de Geografia \\ Universitat de València
}

\section{RESUMEN}

De los bienes que conforman el patrimonio cultural de España, los relacionados con el manejo de los recursos hídricos, ya sean destinados al riego o al abastecimiento humano, han sido históricamente y siguen siendo elementos mínimamente valorados y poco estudiados en el seno de la investigación histórica, artística y etnológica. Sin embargo, estas arquitecturas del agua y conocimientos ancestrales para la gestión de la escasez hídrica juegan un papel importante en la configuración de buena parte de nuestros paisajes; paisajes a los que desde la disciplina geográfica nos tratamos de aproximar. El conocimiento del medio natural, la construcción de artilugios y el uso de determinados artefactos para la captación y distribución del agua son, sin duda alguna, un patrimonio de la sociedad que los ha generado y los ha mantenido. Uno de los elementos más singulares por su antigüedad, concepción técnica y arquitectura son las galerías drenantes o foggaras, un sistema de captación de aguas subterráneas mediante un túnel excavado horizontalmente en el subsuelo. Su identificación, estudio y puesta en valor puede convertirse en un ejercicio efectivo, en primer lugar para la salvaguarda de las galerías como bien patrimonial, en segundo lugar para comprender el origen de un paisaje de regadío y en tercer lugar como estrategia para el desarrollo socioeconómico de un territorio.

Palabras clave: Galerías drenantes, foggaras, minas de agua, patrimonio del agua, paisaje, sistemas de regadío tradicionales, evaluación y puesta en valor del patrimonio del agua.

\section{RÉSUMÉ}

Les biens qui conforment le patrimoine culturel de l'Espagne, ceux en rapport avec le maniement des ressources hydriques, soit destinés à l'irrigation ou à l'approvisionnement humain, ont été historiquement, et sont encore, des éléments faiblement évalués et peu 
étudiés au sein de la recherche historique, artistique et ethnologique. Toutefois, ces architectures de l'eau et connaissances ancestrales pour la gestion de la pénurie hydrique jouent un important rôle dans la configuration d'une bonne partie de nos paysages; Paysages auxquels depuis la discipline géographique il s'agit de se rapprocher. La connaissance du milieu naturel, la construction d'engins et l'utilisation de certains appareils pour le captage et la distribution de l'eau sont, sans aucun doute, un patrimoine de la société qui les a produits et elle les a maintenus. Un des éléments les plus singuliers par son antiquité, conception technique et architecture sont les galeries drainantes, foggaras ou qanats; un système de captage d'eaux souterraines grâce à un tunnel excavé horizontalement dans le sous-sol. Son identification, étude et mise en valeur peut d'abord se transformer en un exercice effectif, en premier lieu pour la sauvegarde des galeries comme bien patrimonial, en deuxième lieu pour comprendre l'origine d'un paysage d'irrigation et en troisième lieu comme stratégie pour le développement socio-économique d'un territoire.

Mots clé: Galeries drainantes, foggara, mines d'eau, patrimoine de l'eau, paysage, systèmes d'irrigation traditionnels, évaluation et mise en valeur du patrimoine de l'eau.

\section{INTRODUCCIÓN}

Los distintos elementos que configuran los sistemas de riego, especialmente las galerías drenantes, constituyen junto a los campos de cultivo un extraordinario patrimonio cultural. Un patrimonio la mayor parte de las ocasiones poco conocido, infravalorado o directamente ignorado. Sin embargo, es por sus históricas implicaciones socioeconómicas y por su capacidad de estructurar paisajes futuros por lo que consideramos oportuno reflexionar acerca de su interés, gestión y, si cabe, optimización. Nos encontramos ante un recurso paisajístico-patrimonial que dispone de una dimensión ambiental y cultural; un bien que necesita la propuesta de acciones orientadas a la investigación, valoración y gestión con fines pedagógicos, ambientales, sociales y económicos.

El análisis de las galerías drenantes en la cuenca hidrográfica del Júcar, se sitúa en el marco de un proyecto de investigación financiado por el Ministerio de Educación y Ciencia ${ }^{1}$. El trabajo nos ha revelado la existencia de varias estructuras subterráneas de captación hídrica, cuyo carácter invita a proponer un tratamiento en profundidad. Muchas son funcionales, poseen un estado de conservación óptimo y un considerable valor patrimonial, por lo que se hace imprescindible iniciar acciones bien de protección, siempre de gestión y cuando sea oportuno de restauración, tanto de la galería como del paisaje del agua asociado. Existen, en la cuenca hidrográfica del Júcar, más de una docena de galerías drenantes que por sus características técnicas, funcionales y patrimoniales merecen un reconocimiento académico y una especial tutela por parte de las autoridades competentes, pero también por parte de los propietarios y agentes locales, para su conservación y puesta en valor.

\section{LAS GALERÍAS DRENANTES, UN PATRIMONIO DESCONOCIDO}

En diferentes textos hemos reflexionado acerca del ensanchamiento conceptual del término patrimonio cultural (Hermosilla e Iranzo, 2004; Hermosilla e Iranzo, 2010). El

1 Proyecto «Las galerías drenantes: estudio, evaluación y revalorización de un elemento singular del patrimonio del agua en la Cuenca Hidrográfica del río Júcar (Teruel, Cuenca, Albacete y Valencia)». Proyecto financiado por la Dirección General de Investigación del Ministerio de Educación y Ciencia. Código HUM200762342 . 
patrimonio, expresión material pero también inmaterial de las actividades humanas sobre un territorio, heredada al tiempo que mantenida y transmitida de generación en generación, ha superado visiones clásicas ligadas a lo grandioso, artístico y monumental. De enfocarse exclusivamente desde una concepción esteticista de lo monumental, el patrimonio ahora también se interpreta de una manera mucho más general, como el lugar de la memoria (Sabaté, 2011). La herencia de lo cotidiano también es patrimonio. Y por tanto son patrimonio los ingenios mecánicos, las tradiciones y las artes populares, las actividades y modos de vida del medio rural, casi siempre modestas si las consideramos aisladas, pero con una fuerza enorme cuando en conjunto dibujan un paisaje urbano, industrial o rural. Esta nueva perspectiva de la cuestión patrimonial enlaza perfectamente con los actuales discursos que, tras una situación ambiental económica, social y cultural insatisfactoria, nos hablan y proponen un uso racional, prudente y equilibrado de los recursos propios.

El patrimonio cultural es, efectivamente, un recurso. Un recurso heredado con un fabuloso potencial, pues refuerza el sentimiento de pertenencia a un territorio y la propia autoestima de una comunidad. El patrimonio pone en valor las prácticas colectivas y la memoria del lugar, contribuye al desarrollo de los grupos sociales y, en combinación con el medio natural, la historia o el propio paisaje, se puede convertir en un dinamizador de la economía local. Cuando un elemento, una técnica o conocimiento es considerado patrimonio cultural incrementamos su valor. Al patrimonializar un bien lo cargamos de sentido, de significado y lo convertimos en representante de su tipología. En definitiva, incrementamos su consideración social y por tanto sus posibilidades de quedar incorporado en la vida cotidiana como una pieza importante, necesaria y asumida.

Y, ¿por qué hablamos de patrimonio y paisajes del agua; o de patrimonio de los regadíos? ¿Son las galerías drenantes, foggaras o minas de agua patrimonio cultural? El medio rural ha sido definido por la Geografía como aquellas áreas caracterizadas morfológica y funcionalmente por las actividades agropecuarias. Aunque en la actualidad se ha producido una difusión de la urbanización y de los modos de vida urbanos sobre los espacios rurales, aún conservamos elementos y paisajes indicativos de unos quehaceres y conocimientos ligados a la actividad agrícola.

Unos de los paisajes rurales más representativos y singulares de la cuenca mediterránea son los paisajes agrícolas de regadío, articulados morfológica, estética y funcionalmente gracias a un rico acervo de artefactos y artilugios destinados al manejo de la escasez hídrica. Efectivamente, en medios donde las precipitaciones son escasas e irregulares y la evapotranspiración, especialmente durante la estación estival, elevada, ha sido imprescindible el desarrollo estrategias que permitieran el aporte extra de agua que los cultivos, ganado y población necesitaban (Laureano, 2001).

Buenos ejemplos de estrategias de gestión del agua, así como de artefactos hidráulicos son hallados en la Península Ibérica. Además, prácticamente desde la Antigüedad y como resultado de la evolución y superposición de saberes de distintos pueblos, la práctica de la agricultura de regadío nos ha dejado buenas muestras materiales e inmateriales de bienes patrimoniales y de paisajes. Paisajes y elementos del patrimonio cultural no siempre conocidos y casi nunca valorados. Como ya hemos descrito en otras ocasiones, las galerías drenantes, foggaras o qanats constituyen el origen de los sistemas de regadío, en aquellas regiones donde las aguas superficiales son escasas e irregulares.

Morfológicamente se presentan como un túnel casi horizontal excavado en el subsuelo o bajo el subálveo de un curso fluvial intermitente, cuya función es extraer las aguas subterráneas al exterior por gravedad (Goblot, 1979; Kobori, 1995). Las tipologías constructivas son variadas y diversas atendiendo al enclave y al periodo de construcción. Cuando las galerías adquieren entidad por la longitud de su trazado se hace imprescindible para 
continuar su construcción y posterior mantenimiento, la apertura de unos pozos verticales que conectan distintos tramos del túnel con la superficie.

Las galerías drenantes, foggaras o qanats cuentan con las cualidades precisas para ser consideradas como patrimonio cultural. Pero ¿cuáles?, ¿todas las galerías? Es evidente que de partida, la singularidad de la captación y el origen de la técnica constructiva nos instan a afirmar que sí. Pero dentro de esta primera patrimonialización académica cabe precisar aún más. Es decir, desnudar al máximo al elemento de manera que se pongan de manifiesto sus principales rasgos, su estado de conservación, su funcionalidad y su reconocimiento social; o dicho de otro modo, su valor. Conociendo el valor real y las características de cada una de las galerías drenantes podremos discernir si pueden ser consideradas en sí mismas como un elemento patrimonial; o lo que es igual, como un bien común con un potencial valor colectivo.

Sin embargo, la patrimonialización de las galerías drenantes cuenta con un hándicap importante. Y es el hecho de tratarse de un tipo de tecnología y estructura en primer lugar poco conocida e invisible para la mayor parte de la población, exceptuando claro está, los paisajes irrigados que genera; y en segundo lugar, que se trata de un artefacto ligado a una sociedad tradicional, relegado por las nuevas técnicas de extracción de agua subterránea, y por tanto considerado como poco operativo, obsoleto y en consecuencia, condenado al olvido y desaparición. Efectivamente, nuestros estudios (Antequera, Iranzo y Hermosilla, 2011) ponen de manifiesto los problemas hidrológicos que presentan las galerías en la actualidad. Sus caudales se han visto reducidos drásticamente por el descenso de los niveles freáticos, lo que ha propiciado su abandono e irremisible destrucción.

Afortunadamente, la singularidad técnica y cultural de las galerías drenantes ha despertado el interés de la academia, que desde distintas disciplinas se ha aventurado a efectuar investigaciones en distintas direcciones. Así hallamos a ingenieros y arquitectos fascinados por las habilidades técnicas de los zanata (técnicos musulmanes especialistas en hidráulica) y de los muqqani (obreros y albañiles encargados de la apertura de las galerías), a hidrogeólogos impresionados por la comprensión de la transmisividad de los materiales del subsuelo, a geógrafos, historiadores y sociólogos interesados en explicar la ordenación paisajística y social resultante de la captación de agua y a arqueólogos preocupados por la datación y el origen de las galerías. Las contribuciones de todos ellos nos han resultado esenciales en las investigaciones que sobre las galerías iniciamos hace ya casi una década.

\section{CAPTACIONES HORIZONTALES EN LA CUENCA DEL RÍO JÚCAR}

\subsection{Caracterización del área de estudio: la Cuenca Hidrográfica del Júcar}

La cuenca hidrográfica del Júcar tiene $21.578 \mathrm{~km}^{2}$ de superficie y se extiende por parte de las provincias de Cuenca, Albacete, Valencia y Teruel. Su espacio geográfico queda conformado por la cuenca del Júcar en sentido estricto y por las subcuencas litorales comprendidas entre la Gola del Saler y el límite de los términos municipales de Cullera y Tavernes de la Valldigna, además de por la cuenca endorreica de Pozohondo. Estamos por tanto, ante un espacio de transición entre el dominio ibérico y el bético, donde podemos diferenciar varias unidades fisiográficas:

A) Sector Ibérico: es una fosa tectónica de gran magnitud desarrollada a partir de la Depresión de Teruel. También comprende la Sierra de Albarracín y la Serranía de Cuenca.

B) Llanura Manchega: es la transición entre el sector Ibérico y el Bético, que tiene el límite en la Sierra de Alcaraz. Su mayor extensión corresponde a los llanos de Al- 
bacete, siendo la meseta de Requena-Utiel su prolongación natural en la provincia de Valencia.

C) Macizo del Caroig: es un espacio de transición entre los relieves Ibéricos y Prebéticos, caracterizado por una amplia plataforma tabular carbonatada y surcada por una red fluvial muy encajada.

D) Sierras y Valles del Prebético interno y externo: son los valles del río Canyoles y del Albaida, en las comarcas de La Costera y la Vall d'Albaida. Se alternan las abruptas sierras carbonatadas del Cretácico con los valles de substratos margosos del Mioceno.

E) Llanura aluvial cuaternaria de La Ribera: está formada por glacis y abanicos aluviales. Los principales rellenos corresponden a los abanicos aluviales de los ríos Magro, Albaida y Sellent, y a la llanura de inundación del río Júcar.

La cuenca posee las condiciones climáticas generales del clima mediterráneo, con un largo periodo árido estival y una acusada irregularidad de las precipitaciones. La mayor parte de la cuenca se sitúa en una zona semiárida. Existen grandes diferencias de temperaturas medias entre las zonas costeras y el interior. Las mayores oscilaciones térmicas se producen en el sector manchego, con una amplitud térmica anual que supera los $20^{\circ} \mathrm{C}$. La media anual de precipitaciones en la cuenca está situada en torno a los $500 \mathrm{~mm}$., que varían entre los $320 \mathrm{~mm}$. de los años más secos hasta los casi $800 \mathrm{~mm}$. de los años más húmedos. Al ser una cuenca extensa existen importantes diferencias espaciales. Los lugares con mayores precipitaciones son el sector de la Sierra de Albarracín, en la cabecera de los ríos Júcar y Cabriel, y la comarca de la Vall d'Albaida. Las zonas más secas se sitúan en la provincia de Albacete y en las comarcas de Requena-Utiel y del Valle de Ayora-Cofrentes.

El río Júcar tiene una longitud de $498 \mathrm{~km}$. Nace en la Sierra de Albarracín, en los Montes Universales, al pie de la Muela de San Felipe, a partir de la confluencia de las aguas de numerosos manantiales. Los recursos totales del sistema ascienden a $2.384 \mathrm{Hm}^{3}$., siendo el caudal medio del Júcar de 49'22 m³/seg. El río tiene en su cabecera un régimen pluvio-nival con un máximo primaveral. En su curso medio y bajo depende más de las precipitaciones estacionales, con un máximo otoñal. Sus principales afluentes son el Guadazaón, el San Martín, el Mira, el Magro, el Canyoles y el Albaida. El curso alto del río, en la Serranía conquense, constituye una meseta rocosa formada por estratos de calizas, margas y yesos. Se alternan los tramos encajados en terrenos calcáreos, con valles amplios y planos sobre terrenos arcillosos.

El curso medio del Júcar se sitúa entre Villalba de la Sierra y el Pantano de Tous. Esta extensa parte media presenta tramos con meandros divagantes y zonas donde el cauce se encaja profundamente. Una vez recibe el agua del Cabriel vuelve a encajarse en el macizo del Caroig. El curso bajo coincide con la llanura aluvial de La Ribera, una zona densamente poblada, con una agricultura intensiva de gran importancia económica. El Júcar recibe ya en la llanura los aportes del Magro y Albaida y describe un recorrido sinuoso con múltiples meandros hasta su desembocadura en la costa de Cullera.

\subsection{Procedimiento para la identificación de las galerías drenantes}

La revisión bibliográfica de las publicaciones existentes en materia de regadíos en el ámbito de estudio, nos ha permitido la identificación de numerosas galerías, que han sido incorporadas a nuestra infraestructura de datos espacial. Esto resulta de gran utilidad para su posterior localización geográfica, imprescindible antes de iniciar la fase de trabajo de campo. Hemos de reseñar la ausencia casi absoluta de información en aquellas publicaciones dedicadas exclusivamente a las galerías drenantes, hallándose la mayor parte de 
las referencias en publicaciones que abordan cuestiones históricas o relacionadas con los regadíos tradicionales.

Asimismo no existe ninguna publicación específica sobre galerías drenantes de la cuenca del Júcar. Además la información sobre las características constructivas y funcionales de los minados en la zona de estudio es muy escasa. En algunos de nuestros trabajos previos sobre la temática, ya se identificaron algunas de las galerías aquí tratadas, destacando la obra «Las galerías drenantes del sureste de la península Ibérica» (Hermosilla, 2006), donde se estudian las galerías de la provincia de Valencia, además de otras del ámbito mediterráneo; o el capítulo dedicado a las foggaras o minas de agua en la publicación de «La Arquitectura del Agua en el Riu Magre: Alcalans-Marquesat»(Iranzo, 2004).

En cuanto a las publicaciones de ámbito local existe bibliografía sobre algún municipio o en ocasiones sobre alguna galería en concreto. Para la comarca de la Vall d'Albaida se han revisado los trabajos de Yago, Jordà y Soler (2004), además de un documento inédito sobre las galerías del término de Bocairent elaborado por Rafael Domenech Domínguez (1994). En la comarca de la Plana de Requena-Utiel destacan las publicaciones de Jorge Hermosilla sobre el regadío histórico, editadas en 2002 y 2005. Por lo que respecta al municipio albaceteño de Alpera debemos reseñar los estudios de Vicente Taroncher (1984a y 1984b), tanto sobre la captación de las aguas de Alpera, como sobre la pugna que por dicho agua sostuvieron durante varios siglos, los municipios de Alpera y Almansa. Este conflicto también se encuentra bien documentado en las publicaciones de Pereda Hernández (1987), quien ha realizado con posterioridad estudios sobre el Pantano de Almansa y la Comunidad de Regantes homónima, en 1992 y 1996 respectivamente.

En el área de estudio se han identificado 132 galerías, de las cuales hemos inventariado y catalogado un total de 77 minados, lo que supone casi el $60 \%$ de las captaciones localizadas. Las galerías identificadas pero no incorporadas al inventario no se han analizado con detalle ya que, o bien la accesibilidad a las mismas estaba muy limitada por factores múltiples, o bien han quedado desmanteladas por algún tipo de transformación de los usos. La cartografía de las galerías ha resultado una fase esencial de la investigación. Se ha recurrido, además de a las fuentes bibliográficas, a cartografía histórica y a la fuente oral para la localización de las captaciones. Esta labor se ha llevado a cabo tanto en gabinete como durante el propio trabajo de campo.

\subsection{Metodología de evaluación de las galerías drenantes del área de estudio}

En nuestro deseo de urdir la valorización de este singular patrimonio hidráulico y de sus paisajes asociados, resulta imprescindible su caracterización y evaluación. Sólo mediante el diagnóstico elaborado, a partir de este análisis integrado, estaremos en disposición de formular propuestas de acción, coherentes y oportunas. Para la valoración patrimonial de las galerías drenantes hemos utilizado una metodología de evaluación semicuantitativa de tipo multicriterio. Se fundamenta en la definición de diez criterios o indicadores que son valorados para cada una de las captaciones. Si un criterio se cumple se valora como 1. En caso contrario, 0 . Por lo tanto una galería puede alcanzar una valoración máxima de 10 puntos.

La selección de criterios o indicadores para determinar el valor patrimonial de cada galería es resultado de la experiencia acumulada por la unidad de investigación ESTEPA (Estudios del Territorio, Paisaje y Patrimonio) en el estudio del patrimonio del agua. Dichos criterios tratan de considerar tanto aspectos de tipo estructural, como aspectos de tipo funcional, territorial, histórico, y social. En este sentido, los criterios o indicadores utilizados son:

1. Cultura del agua: hace referencia a la relación que posee la obra o artefacto hidráulico con el uso tradicional del agua en la localidad. 
2. Representatividad: indica si el artefacto u obra hidráulica reúne rasgos comunes, desde el punto de vista de la funcionalidad y morfología, a otros artefactos existentes en la zona de la misma tipología patrimonial.

3. Autenticidad: define el grado de fidelidad que posee el artefacto hidráulico en relación a su configuración original.

4. Integridad: señala el estado de conservación del elemento o artefacto hidráulico y el grado de funcionalidad que posee.

5. Tecnología: se considera el ingenio en la técnica utilizada, la innovación y la mejora que el artefacto introduce en el sistema socioeconómico del área de influencia.

6. Artístico: hace referencia al grado de desarrollo creativo y ornamental que alcanza el artefacto u obra hidráulica como resultado de la aplicación de unas técnicas y al uso de determinados materiales «nobles».

7. Territorial: indica el grado de interacción entre la obra o artefacto hidráulico y el entorno en que se sitúa, tanto por su visibilidad paisajística como por su armonía con el entorno.

8. Histórico-social: se valoran aspectos como su antigüedad, la disponibilidad de documentación histórica y cartográfica que refrende dicha antigüedad y el grado de conocimiento e identificación de la población con el artefacto hidráulico.

9. Hidráulico: en este criterio se considera si el artefacto forma parte constituyente de un sistema de regadío tradicional de relevancia, así como las dimensiones e importancia del artefacto hidráulico en relación con el conjunto del sistema de regadío.

10. Participación y concienciación social: hace referencia al nivel de implicación de los agentes sociales en la protección, conservación y puesta en valor del patrimonio del agua

Como arriba apuntábamos, la evaluación de las galerías drenantes se establece a partir de la puntuación numérica de los diez criterios. El trabajo de campo es esencial a la hora de hacer efectiva la aplicación del método de evaluación. Algunos de los indicadores o criterios definidos en la metodología necesitan de la observación directa para su correcto tratamiento.

\subsection{Jerarquización de las galerías en función de su valor patrimonial}

Merced a esta metodología de evaluación se ha establecido una jerarquización de las galerías inventariadas. Dicha información se convierte en esencial en el proceso de reflexión sobre las acciones más oportunas para la gestión adecuada del bien patrimonial. La jerarquía resultante nos permite la formulación de medidas de conservación, gestión y actuación en función del valor que han obtenido en la metodología de evaluación. En las galerías de menor valoración las medidas a implementar no suelen ir más allá de la localización geográfica, breve caracterización y catalogación. Muchas de éstas han quedado fuera de uso al perder su caudal, mientras que otras, debido al abandono de la actividad agrícola y despoblamiento rural se han ido deteriorando por falta de mantenimiento, llegando incluso a su colapso.

Es por ello por lo que los mayores esfuerzos han sido centrados en la puesta en valor de aquellas galerías cuyas notables cualidades así lo sugieren. En la tabla 1 mostramos un resumen de los resultados obtenidos, tras implementar el método de evaluación en las 77 galerías catalogadas. Los resultados señalan 14 galerías con un valor patrimonial alto y muy alto; y 11 galerías con una valoración baja o muy baja. 
Tabla 1

LAS GALERÍAS DRENANTES INVENTARIADAS CON MEJOR Y PEOR VALORACIÓN PATRIMONIAL

\begin{tabular}{|c|c|c|}
\hline $\begin{array}{l}\text { VALORACIÓN } \\
\text { PATRIMONIAL } \\
\quad(0-10)\end{array}$ & GALERÍA & $\begin{array}{l}\text { MUNICIPIO } \\
\text { (PROVINCIA) }\end{array}$ \\
\hline \multirow{6}{*}{10} & Font del Port & Albaida (Valencia) \\
\hline & $\begin{array}{c}\text { Mina de Nuestra Señora de la Asunción } \\
\text { o de Monserrat }\end{array}$ & Monserrat (Valencia) \\
\hline & Font d'Elca o de l'Arcà & Salem (Valencia) \\
\hline & Mina Aguas de Zucaña & Almansa (Albacete) \\
\hline & Fuente del Porvenir & Almansa (Albacete) \\
\hline & Fuente del Canto & Cuenca (Cuenca) \\
\hline \multirow{3}{*}{9} & Mina de la Huerta Nueva & Buñol (Valencia) \\
\hline & Mina de la Casica Cantos & Almansa (Albacete) \\
\hline & Mina de Aguas Nuevas & Almansa (Albacete) \\
\hline \multirow{5}{*}{8} & Mina de Santa Bárbara o del Teular & Bocairent (Valencia) \\
\hline & Mina del Abrullador & Chella (Valencia) \\
\hline & Mina de la Huerta Vieja & Quesa (Valencia) \\
\hline & Fuente Redonda & Alpera (Albacete) \\
\hline & Mina de la Casa Martínez 1 & Almansa (Albacete) \\
\hline- & --- & --- \\
\hline \multirow{5}{*}{2} & Cava de la Boleta & Benigànim (Valencia) \\
\hline & Alcavor Font dels Alborets & Bocairent (Valencia) \\
\hline & Mina Casa de la Matea & Enguera (Valencia) \\
\hline & Mineta de la Marjal & Monserrat (Valencia) \\
\hline & Mina del Pouet & Montroi (Valencia) \\
\hline \multirow{3}{*}{1} & Mina del barranco de la Mora & Ayora (Valencia) \\
\hline & Cava de la Casa Roja & Bellús (Valencia) \\
\hline & Alcavor i bassa del Mig dels Alborets & Bocairent (Valencia) \\
\hline \multirow{3}{*}{0} & Fuente del Lobo & Ayora (Valencia) \\
\hline & Cava de l'Albelló o de Corfa & Llutxent (Valencia) \\
\hline & Galería de la Casa Blanca & Requena (Valencia) \\
\hline
\end{tabular}


Entre las galerías mejor valoradas, con una puntuación entre 8 y 10 puntos según los criterios de nuestra metodología de evaluación patrimonial, se observa la presencia de hasta seis minados pertenecientes al municipio de Almansa (Ver figura 1). Otras galerías que alcanzan una buena puntuación se localizan en la provincia de Valencia, aunque algo más dispersas geográficamente: Salem, Albaida, Buñol, Bocairent, Chella y Quesa. La concentración geográfica de galerías evaluadas como de alta calidad en Almansa y Alpera justifica que efectuemos una propuesta central de puesta en valor en estos municipios manchegos. Además, como seguidamente comentaremos, estas localidades cuentan con otros elementos del patrimonio del agua, que articulados entre sí nos ayudan a comprender su organización territorial, paisajística y económica, pasada y cuanto menos presente.

\section{PROPUESTA METODOLÓGICA PARA LA PUESTA EN VALOR DE LAS GA- LERÍAS DRENANTES}

\subsection{Introducción}

Las actuaciones sobre el patrimonio cultural pueden estar dirigidas bien hacia la protección y conservación, bien hacia la gestión o bien hacia la rehabilitación de sus elementos. En todo caso, hemos de considerar siempre la gestión como tarea fundamental en todo proceso de puesta en valor territorial. La gestión del patrimonio y del paisaje asociado entraña mucha complejidad. Requiere de un enfoque interdisciplinar y del consenso con los agentes locales, que serán en última instancia los artífices del éxito o fracaso del pro-

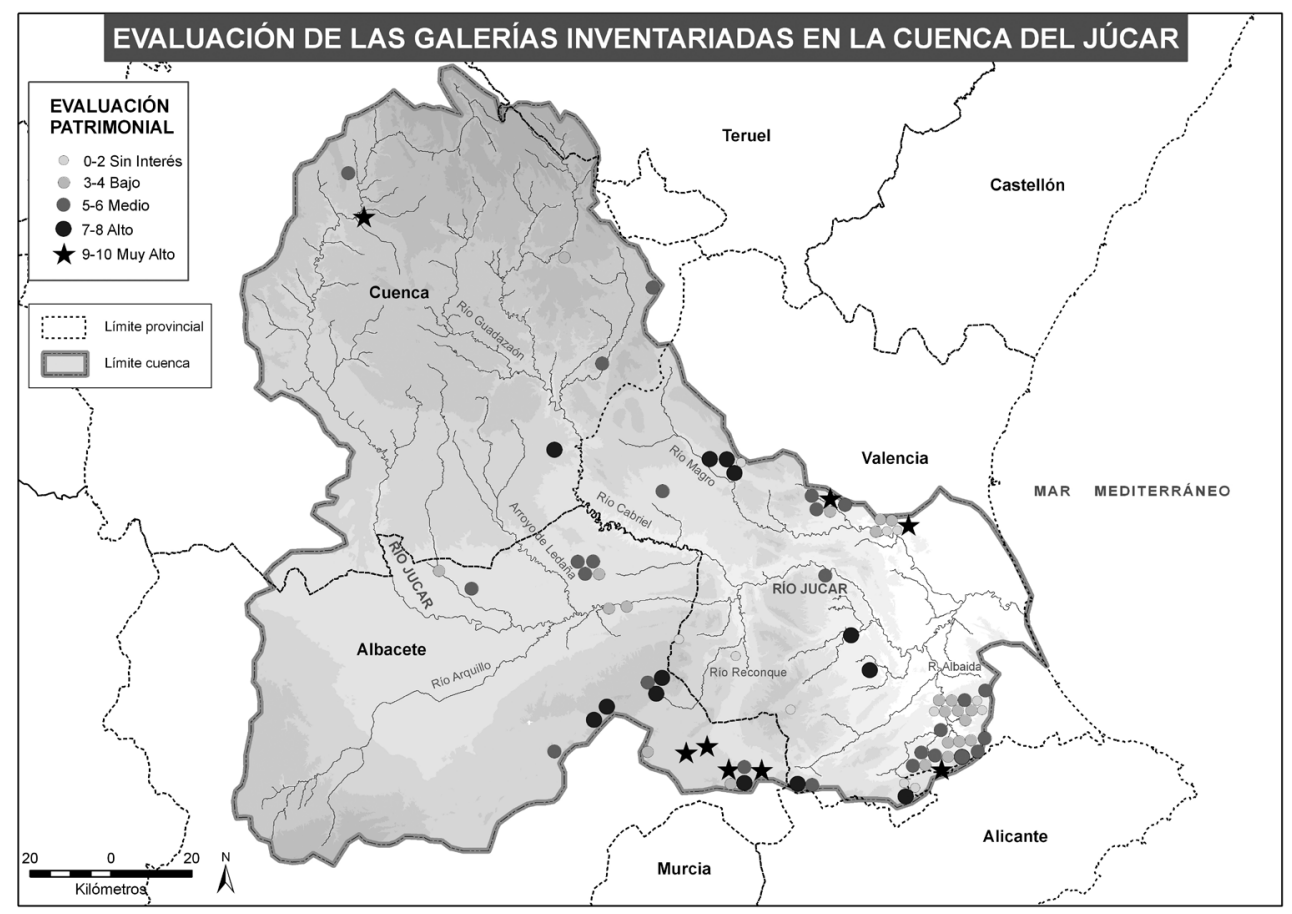

FIGURA 1. Mapa de localización y evaluación de galerías drenantes en la Cuenca Hidrográfica del Júcar. 
yecto de puesta en valor. Para el caso que nos ocupa, la planificación de acciones ha de tener cierto carácter estratégico. Se trata de gestionar un tipo de bienes que conforman una estructura paisajística singular, de manera que se mantenga la sostenibilidad y el sentido de identidad. El patrimonio y el paisaje son un recurso, pero esta percepción ha de impregnar a la población local. Es por ello por lo que una de las primeras medidas de todo proyecto de puesta en valor del patrimonio ha de referirse a la sensibilización y difusión de las cualidades de los bienes. El reconocimiento propio de estos bienes por parte de la población local es por tanto imprescindible para asegurar el éxito de la acción.

Las propuestas de actuación sobre el patrimonio hidráulico de las galerías drenantes no han de caracterizarse exclusivamente por la conservación y rehabilitación de los túneles, sino que deben ir más allá. Estas estructuras de captación de agua subterránea no son elementos aislados. Han de ponerse en valor relacionándolas con el espacio geográfico que las determina y con el paisaje que generan. También con el conocimiento tradicional que entraña su construcción y mantenimiento, con la gestión comunitaria del agua y con la diversidad de usos que ésta tiene. En definitiva, en comunión con el acervo de bienes materiales e inmateriales que constituyen el conjunto hidráulico (Gil, Martínez y Gómez, 2010). No obstante, en muchas ocasiones sus cualidades no son reconocidas, generalmente por la falta de autoestima de los agricultores con respecto a sus actividades, o bien sencillamente por desconocimiento del valor cultural que galerías, red de acequias, gestión del agua y paisaje agrícola poseen.

La optimización de estos recursos patrimoniales implica, en primera instancia, la aplicación de un método de análisis paisajístico-patrimonial integrado, que revele el funcionamiento intrínseco del sistema. Definir la excepcionalidad del conjunto patrimonial que se desea poner en valor, implica reconocer a través de la investigación geográfica las relaciones entre los bienes patrimoniales y la evolución de los procesos que han organizado el espacio que los alberga. O dicho de otro modo, necesitamos efectuar una lectura en profundidad, del presente y del pasado, de las relaciones que se establecen entre el territorio y la sociedad que lo habita y lo transforma. En segunda instancia, también es necesario definir un proyecto. Una iniciativa formulada en clave de plan estratégico que concrete los objetivos, canalice los recursos tanto humanos como económicos, programe los esfuerzos y materialice las actuaciones de puesta en valor paisajístico-patrimonial.

Es por ello por lo que, para el caso que nos ocupa, hemos iniciado nuestra labor con esa primera fase principalmente ligada al diagnóstico territorial. Nuestra investigación sobre galerías drenantes y regadíos en la cuenca del Júcar nos ha permitido localizar y analizar los mejores sistemas; unos sistemas conformados no sólo por estos singulares artefactos de captación hídrica, sino por el conjunto constituido por galerías, red de canales y acequias, elementos de acumulación del agua, artefactos hidráulicos protoindustriales y parcelario agrícola. Después de la localización e identificación de las galerías drenantes, así como del resto de componentes del sistema tradicional de riego, se han preparado fichas de caracterización de todos ellos. Aunque en el presente artículo no nos vamos a concentrar en la fase de caracterización seguida, merece la pena indicar que la información tratada ha sido útil en la caracterización y evaluación de los paisajes. De esta manera, con los materiales incorporados en el sistema de información geográfica (SIG), estamos en disposición de implementar nuestra metodología de evaluación del patrimonio hidráulico, cuyos resultados son determinantes a la hora de orientar la puesta en valor de las galerías drenantes.

Los resultados de la evaluación, mostrados en la tabla nos instan a dirigir las medidas de actuación a la difusión y, donde sea apropiado, a la recuperación de este rico patrimonio cultural y paisajístico. Seguidamente presentamos de manera más concreta y sistemática cómo plantear algunas estrategias de puesta en valor de las galerías drenantes. Reiteramos, 
no es conveniente tratar exclusivamente a las galerías de captación como un elemento aislado, sino que es más adecuado considerar también al sistema de regadío y/o abastecimiento asociado, con los diferentes elementos que lo componen: acequias, partidores, balsas, acueductos, sifones, molinos hidráulicos, parcelario, tipo de cultivos etc. Así, efectuamos un tratamiento más integral de un conjunto paisajístico-patrimonial.

\subsection{La puesta en valor: el Parque Patrimonial de las Galerías de Almansa-Alpera}

La puesta en valor del patrimonio y sus paisajes exige reflexión, método y consenso. Para emprender acciones directas y concretas hemos de conocer sus rasgos, cualidades y hacer que estos elementos hidráulicos se reconozcan como patrimonio. Aunque el proyecto en el que se enmarca este artículo ha dirigido nuestros esfuerzos hacia las fases de identificación, caracterización y evaluación de las galerías, apuntamos aquí algunas acciones concretas de puesta en valor, y desarrollamos la propuesta de parque patrimonial para el conjunto de galerías drenantes de Almansa-Alpera.

Como parte de un Plan de Acción Territorial o de un Plan Estratégico Regional, Comarcal o Local, las posibles iniciativas de promoción de los recursos paisajístico-patrimoniales han de plantearse como proyectos concretos, que serán estructurados a través de distintas actuaciones (Palenzuela, Cobacho y Guzmán, 2000). Un proyecto de puesta en valor del patrimonio y paisaje del agua ha de ser coherente y claro, de manera que facilite las acciones previstas a los gestores del mismo, al tiempo que propicia la comprensión de la iniciativa por parte de la ciudadanía e instituciones externas.

En este sentido consideramos que un proyecto de puesta en valor del patrimonio y paisaje del agua ha de contar con los siguientes elementos: un título que visualice el objeto de la propuesta; una justificación del proyecto que refrende las posibilidades del proyecto a partir de experiencias similares; unos objetivos donde se señale las metas que pretendemos alcanzar; un equipo gestor compuesto por técnicos y otros agentes interesados (administración, propietarios del bien, entidades externas...), encargado de marcar los tiempos y buscar los medios para la materialización del proyecto; un análisis de los potenciales usuarios de modo que la actuación trate de cubrir de la mejor manera posible sus expectativas; unas actuaciones específicas que concreten con más detalle las acciones a desarrollar, así como las tareas asociadas; y una agenda de proyecto que defina el calendario de ejecución de cada una de las fases de la iniciativa.

El proyecto que proponemos es el diseño de un parque patrimonial articulado en torno a las galerías drenantes de Almansa-Alpera. Definimos parque patrimonial como una estrategia de desarrollo territorial basada en la construcción de una estructura lúdico-cultural basada en una serie de recursos patrimoniales y paisajísticos. Un parque patrimonial es una iniciativa que tiene un doble objetivo: promover la preservación del patrimonio cultural de un área geográfica, y favorecer el desarrollo socioeconómico de la misma (Sabaté, 2005; Hermosilla e Iranzo, 2009). El parque patrimonial tiene como principales características:

1. Son lugares o territorios donde se privilegia el esparcimiento a través del uso de unos recursos culturales y naturales en equilibrio.

2. Son ambientes humanizados y vivos, donde conjuga la recreación con el habitar.

3. No es ni un parque natural ni un parque temático. Ofrece un tipo de servicio íntimamente ligado a la identidad cultural de un territorio.

4. Es un espacio donde el patrimonio y otros recursos territoriales, culturales, históricos y escénicos se combinan para formar un paisaje acordado, que cuenta la historia de dicho territorio. 
5. Un parque patrimonial representa un espacio donde los recursos naturales y culturales son claves en el desarrollo económico.

Por tanto, los cuatro pilares sobre los que se sustenta un parque patrimonial son: preservación de los recursos patrimoniales, educación, ocio y desarrollo socioeconómico.

\subsubsection{Los recursos patrimoniales, la historia y el contexto geográfico del área de estudio}

En el municipio de Alpera las galerías de «Fuente Redonda», «las Fuentes» y la «Fuente de las Dos Hermanas» originan la acequia de Aguas de Alpera. En término de Alpera este canal abastecía en su recorrido nueve artefactos hidráulicos, además de emplearse para el regadío de las tierras adyacentes al casco urbano. De los nueve molinos existentes el edificio se encuentra en un buen estado de conservación en los molinos de San Antonio o José Bueno, de Tobillos, de la Villa, de Juan José Sanz o Primero y en la Fábrica de Luz «La Máquina». Mención especial merece el Molino de San Gregorio, ya que al buen estado de la edificación se añade la conservación prácticamente intacta de la maquinaria. Este artefacto fue el que tuvo un funcionamiento más longevo en el municipio, ya que dejó de moler en 1999. En la actualidad ha sido adquirido por la Diputación Provincial de Albacete, con la intención de restaurarlo e instalar allí un museo.

La «Mina de Aguas Nuevas» de Almansa se emplea como complemento de la acequia Aguas de Alpera. Una vez el caudal es derivado al exterior se une al canal antedicho, cuyas aguas desembocan en el Embalse de Almansa. Éste, proyectado y construido en el año 1584, es el más antiguo de Europa en funcionamiento. Desde el embalse parte una acequia, en buen estado pero actualmente fuera de uso, que bonifica las tierras de la partida de El Hondo, hasta casi alcanzar el término municipal de Ayora. El Embalse de Almansa es un recurso ambiental y cultural de primer orden. Alberga valores de tipo natural o ecológicos, pero también paisajísticos, históricos, técnicos y socioeconómicos que no debemos obviar.

Otra galería que genera un sistema hidráulico de gran interés es la Mina de Aguas de Zucaña. Una vez que el caudal de la galería sale al exterior, suministra a una acequia situada en su primer tramo, sobre la rambla de los Molinos. La acequia abastecía en el pasado a nueve artefactos hidráulicos, antes de alimentar a las Balsas del Concejo (Nueva y Vieja). Desde ellas, plenamente funcionales en la actualidad, se distribuye el agua a la zona de regadío de la Vega de Almansa. En uno de los molinos abastecidos por la acequia, concretamente en el Molino Alto, el Ayuntamiento de Almansa ha llevado a término acciones para su recuperación y utiliza el edificio como Aula de la Naturaleza

Aunque no llegó a cubrir las expectativas de los agricultores que apostaron por su apertura, también contamos con otra galería drenante: la «Fuente del Porvenir», de la que se conservan las lumbreras y restos de la acequia que debía de alumbrar las huertas al noroeste del casco urbano de Almansa. Finalmente, en la Sierra de Almansa también tenemos dos magníficos ejemplos de galerías al disponer de los minados de la Casica Cantos y los de la Casa Martínez, que en ambos casos son de propiedad particular.

\subsubsection{El proyecto de puesta en valor}

La propuesta de Parque Patrimonial de las Galerías de Almansa-Alpera pretende articular un escenario lúdico-educativo con un hilo conductor: el paisaje y el patrimonio del agua. El parque patrimonial se convierte, por una parte, en un recurso territorial en sí mismo pues actúa como motor del desarrollo local; y, por otra parte, en el argumento oportuno para la preservación, recuperación y reconocimiento de un patrimonio tan poco valorado como el de los regadíos y gestión del agua. El agua es un elemento indispensable para la 
vida y progreso de las sociedades. La humanidad ha generado toda una cultura del agua, especialmente en el interior peninsular, donde el clima mediterráneo continentalizado incorpora rasgos de aridez. La ausencia de agua disponible durante algunas estaciones del año, y especialmente su escasez durante los periodos de máximas temperaturas y fuerte evapotranspiración, han agudizado el ingenio humano para su captación y aprovechamiento. Un buen ejemplo lo hallamos en las localidades albaceteñas de Alpera y Almansa. Sus habitantes han utilizando estrategias diversas para captar y utilizar el recurso agua y gestionar dicha escasez; de manera que podemos identificar un agregado de elementos materiales e inmateriales (conocimientos) sobre los que organizar un discurso interpretativo.

Como arriba se apuntaba, el conjunto Alpera y Almansa tiene unos interesantes ejemplos de captación del agua a través de galerías drenantes o foggaras, de distribución mediante canales y acequias, y de uso de la misma, ya sea en la actividad agrícola, como fuerza motriz en artefactos protoindustriales (molinos) o en el abastecimiento de la población. Lamentablemente, este patrimonio tan cotidiano y de aparente escaso interés, está experimentando un rápido deterioro por desuso. Además se trata de una infraestructura humana poco conocida y reconocida y, por tanto, poco valorada como bien cultural. En definitiva, un conjunto, sin duda patrimonial, infravalorado por extraños pero también por buena parte de los propios del lugar.

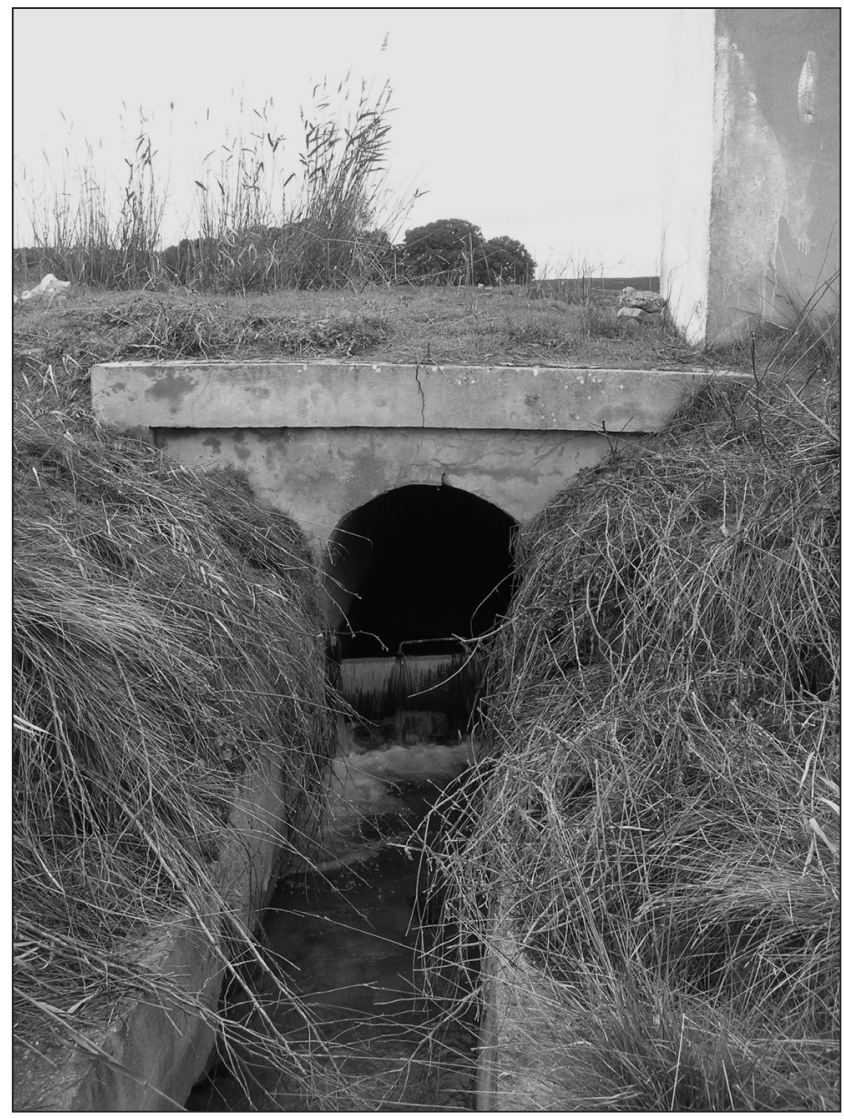

Figura 2. Bocamina de la fuente de Dos Hermanas. 
Al proponer el diseño del Parque Patrimonial de las Galerías de Almansa-Alpera pretendemos recuperar esa cultura y arquitectura del agua, que dejó su huella sobre el paisaje, de manera que se convierta en una pieza interesante del desarrollo socioeconómico del área. El parque se materializa a modo centro de interpretación al aire libre, donde a través de dos recorridos señalizados y teleguiados se descodifica a los visitantes el patrimonio y paisaje del agua. Existen, por tanto, dos itinerarios propuestos. El primero tiene como origen las galerías de Fuente Redonda y de Fuente de las Dos Hermanas, y la mina de las Fuentes, las tres en el término municipal de Alpera. Se tratan de tres galerías de captación de agua subterránea, que abastecen a una acequia, la cual conduce el agua hasta el histórico embalse de Almansa. Las galerías dejan su huella en el paisaje, no sólo en la bocamina, sino también en la superficie cuando se percibe la sucesión de montículos dispuestos linealmente durante varios kilómetros. Nos estamos refiriendo a las lumbreras o pozos de aireación. Estos elementos también pueden ser considerados recursos patrimoniales (Gómez et al., 2009).

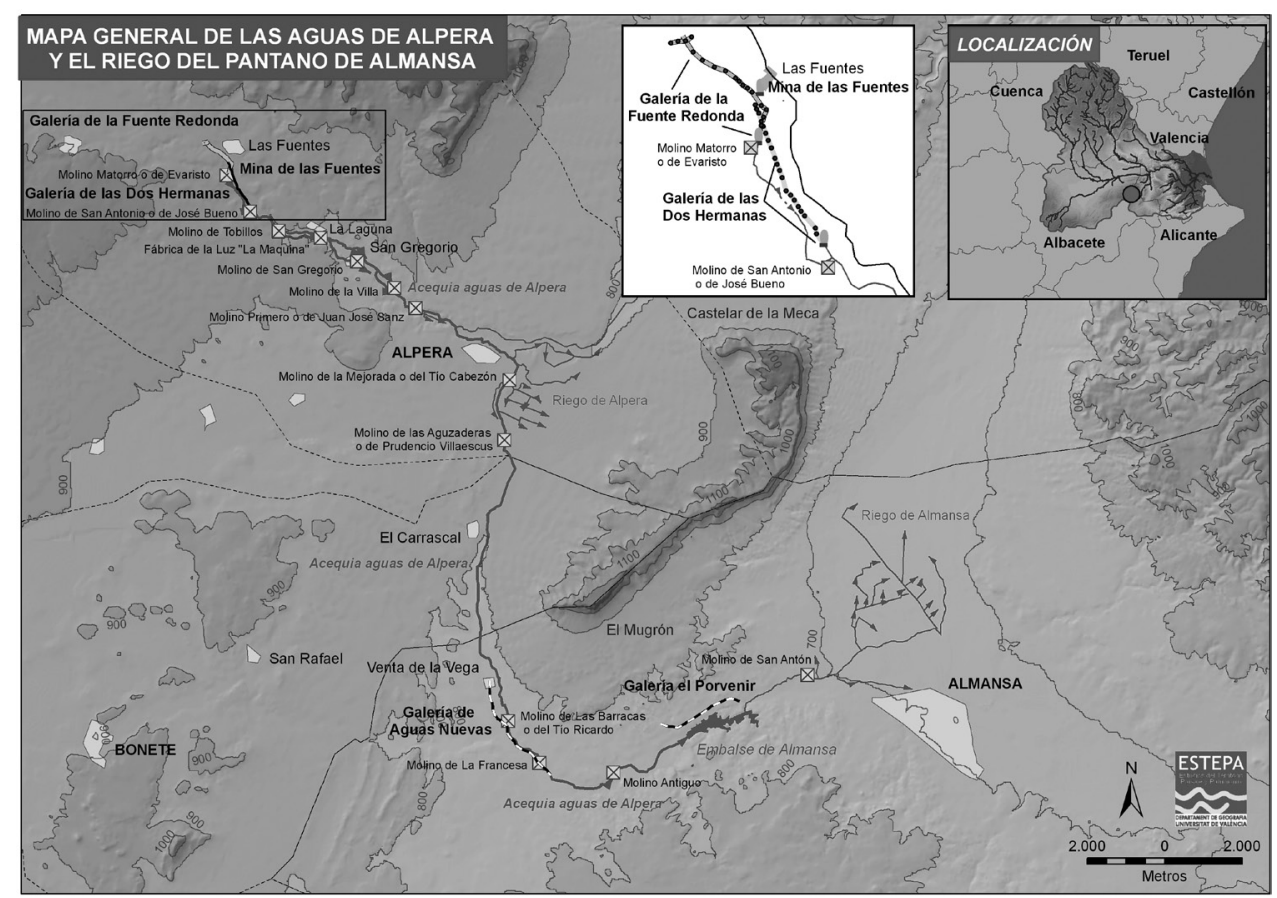

FIGURA 3. Sistema de riego de Alpera-Almansa propuesto en el diseño de parque patrimonial.

El primer itinerario sigue el trayecto que, desde el término municipal de Alpera, realizan las aguas destinadas a regar la partida del Hondo en Almansa. La acequia parte desde Fuente Redonda aunque recoge también las aguas de la Mina de las Fuentes y de la Fuente de las Dos Hermanas. Tras propulsar la maquinaria de siete molinos harineros alcanza, suministrando en el pasado agua a la población de Alpera. Tras superar el núcleo urbano de Alpera, dos molinos más eran abastecidos con agua de la acequia, la cual sigue su trazado en dirección sur, hacia el término municipal de Almansa. En su recorrido, antes de recibir los aportes de otra galería, la Mina de Aguas Nuevas, abastecía a dos molinos más. Las 
cuatro captaciones de agua subterránea citadas fueron construidas entre los siglos XIX y principios del XX. En el caso de la última, se excavó expresamente entre 1821 y 1832, para incrementar la dotación de aguas del Embalse de Almansa. Efectivamente, tras recibir los aportes de la Mina de Aguas Nuevas, itinerario y acequia continúan su recorrido. La acequia abastece a un molino harinero y finalmente desagua en el embalse de Almansa. Las aguas del embalse han tenido históricamente gran importancia económica, ambiental y paisajística, ya que sirvieron para crear un paisaje agrícola de regadío, de más de 1.700 hectáreas, en la partida conocida como el Hondo. Antes de alcanzar la zona de riego, la acequia procedente del embalse abastecía al molino de San Antón.

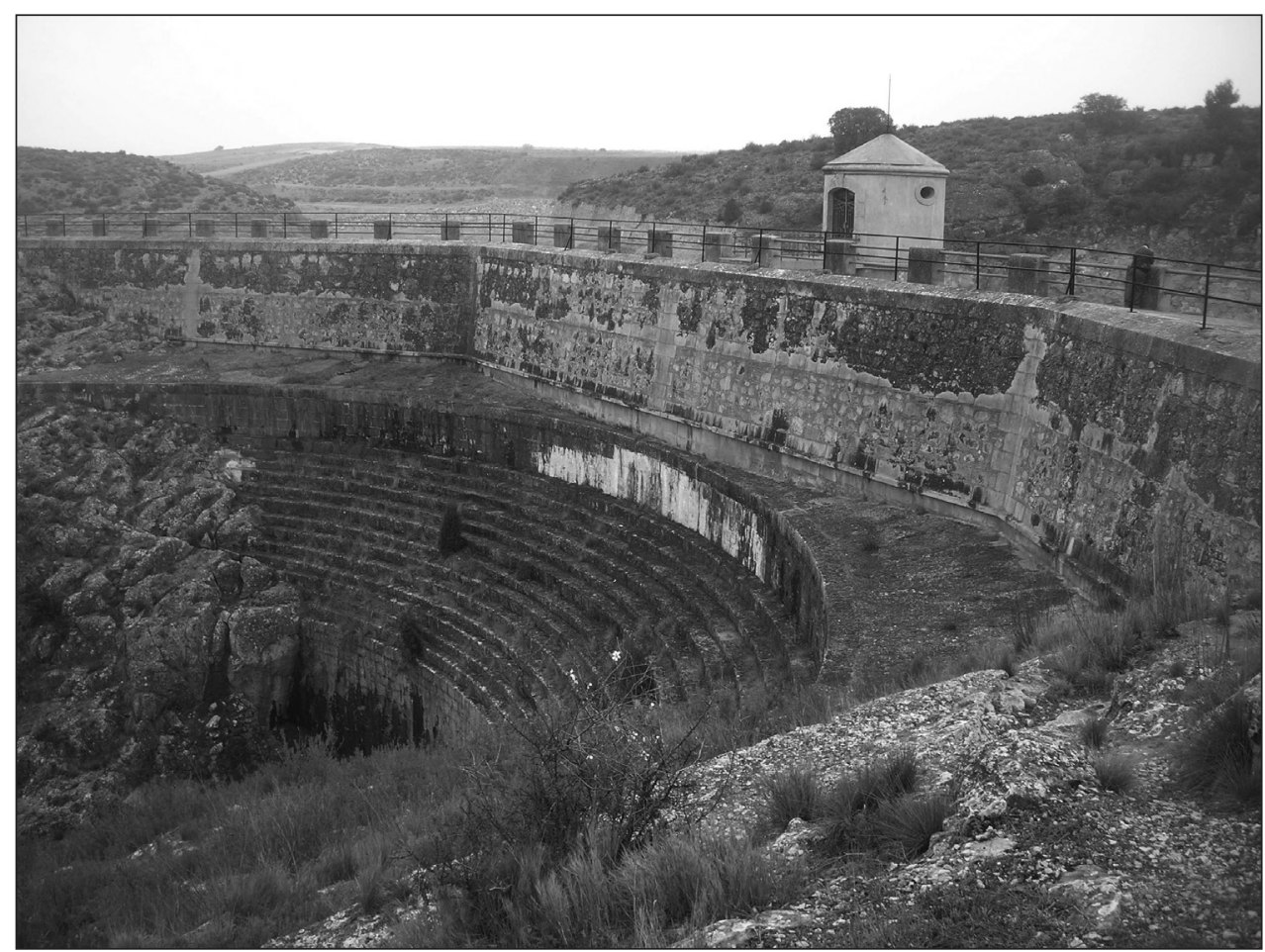

FIgURA 4. Presa del Embalse de Almansa.

El segundo sector que conforma el parque patrimonial corresponde al sistema hidráulico de la Mina de Agua de Zucaña, localizado al sur del casco urbano de Almansa, en el entorno del barranco de los Molinos. Tanto la galería como el conjunto hidráulico constituido por acequia, balsas, molinos y huertos consta de notable interés por su antigüedad (galería de posibles orígenes bajo medievales), por sus características constructivas y arquitectura, por el entorno natural, por la relevancia industrial (nueve molinos harineros se sucedían escalonados siguiendo la acequia) y por su importancia económica, pues las aguas alumbraban las antiguas huertas de Almansa. Una propuesta posible sería la restauración o la inserción dentro de alguna ruta, de alguno de los molinos que aún poseen la edificación. Sería el caso del Molino de los Álamos, cuyo edificio se halla, en parte, en estado de ruina; del Molino de la Torre, con un buen estado de conservación aunque de propiedad particular; 

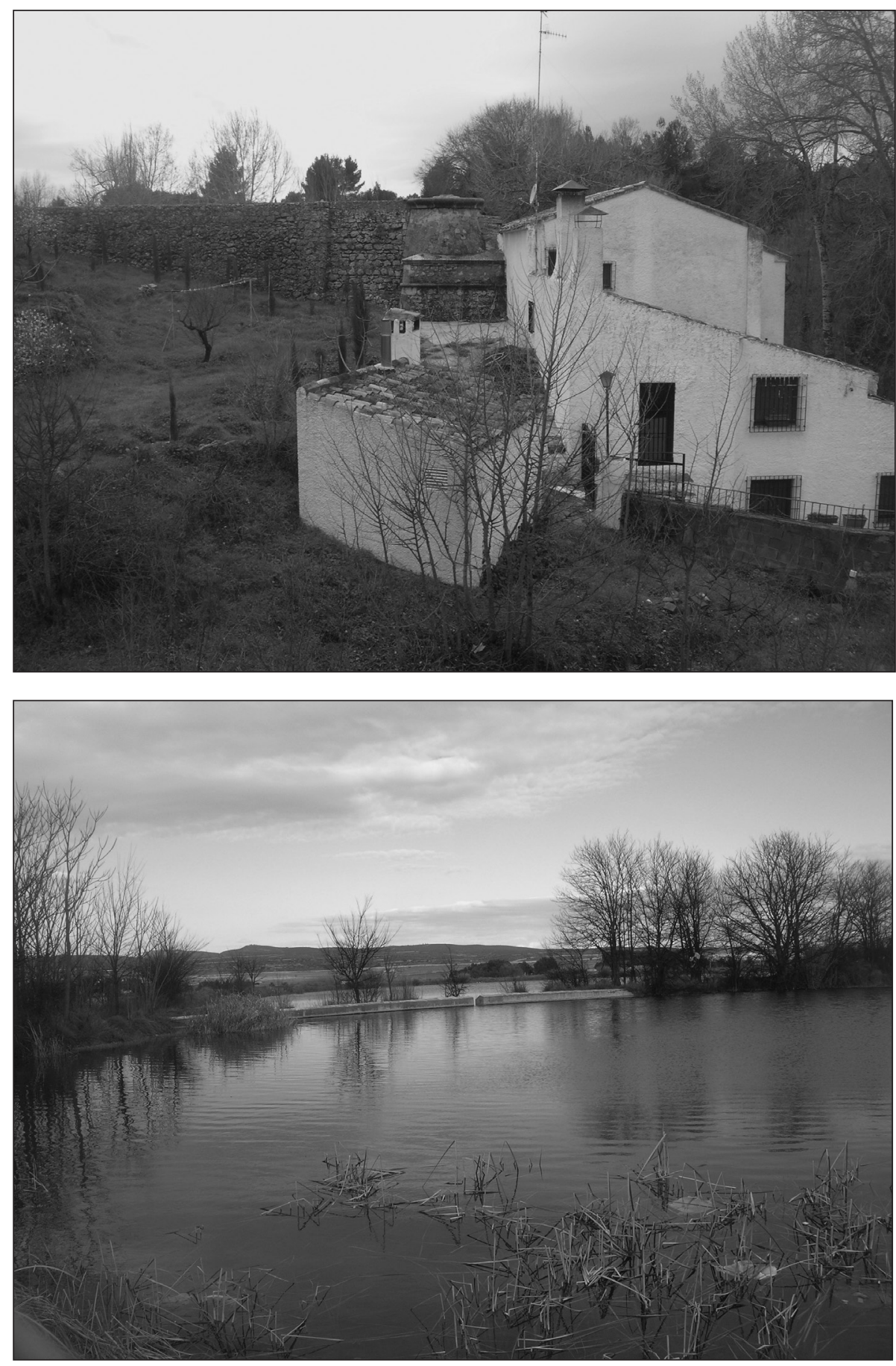

Figura 5. Molino harinero y Balsa Nueva y Vieja del Concejo. 
del Molino de las Monjas, con un estado de conservación deficiente; y del Molino de la Balsa, del que sólo permanece en buen estado la alberca, estando el edificio en ruinas.

Definido y caracterizado el ámbito del parque con el agua procedente de galerías drenantes como hilo argumental, es el momento de que sus gestores lo doten de contenidos, aprovechen cada recorrido aquí propuesto para explicar una historia, continúen con las labores de investigación y documentación sobre el patrimonio del parque, impliquen a la población, técnicos, administraciones y asociaciones locales en la iniciativa, busquen ayudas económicas y subvenciones, y empiecen a definir algunas actuaciones concretas de materialización del parque.

De acuerdo con los apuntes que Gil, Martínez y Gómez (2010) hacen para un proyecto similar en Pliego (Murcia), la propuesta de parque patrimonial de las galerías de AlperaAlmansa no deja de ser la revitalización de un sistema paisajístico territorializado, en el que naturaleza y cultura comulgan como parte de un concepto único, patrimonio (Sabaté, 2005: 32). Así, al incorporar las raíces etnográficas, los bienes tangibles y los bienes intangibles dentro de las políticas de desarrollo local y planificación estratégica, conseguimos que éstos sean percibidos como recursos susceptibles de revertir positivamente a nivel económico, pero también identitario, pedagógico, turístico y por supuesto ambiental.

\section{CONCLUSIONES}

Aunque en la actualidad el medio rural es considerado como un espacio plurifuncional, su estructura y aspecto aún conservan la influencia de un pasado agropastoril y silvícola: usos extensivos de la tierra, pequeños núcleos de población y asentamientos dispersos cuya arquitectura está totalmente integrada en el paisaje, y un modo de vida basado en el uso racional de los recursos próximos. Lejos de entender su configuración y dinámica como una debilidad, la percepción de sus recursos como una fortaleza pueden convertirlo en un espacio de oportunidad tanto para los que lo habitan como para los que lo visitan. El resultado de esta interacción hombre-medio es un mosaico de componentes a los que denominamos bienes, que manifiestan unos modos de vida ligados al territorio. Estos bienes, naturales y culturales, que conforman el sistema patrimonial local y que se manifiestan espacial y visualmente a través de los paisajes culturales, desempeñan una función social, en tanto en cuanto ayudan a la población a reconocer en ellos sus señas de identidad, y sirven de factor de desarrollo a la comunidad a la que pertenecen.

En este sentido, nos parece oportuno experimentar nuevas formas de valorizar el paisaje y el patrimonio cultural del medio rural con el múltiple objetivo de activar el tejido económico y social, a través de la creación de empleo en primer lugar, y en segundo lugar fomentar nuevos modos de relación e interacción entre los agentes locales. Los bienes naturales y culturales, integrados en el paisaje y patrimonio, han pasado a ser una exigencia social. Su disfrute supone una fuente de ingresos para aquellos agentes locales, públicos y privados, que sepan efectuar un correcto uso y gestión de los mismos. Existen experiencias que demuestran que, tanto el paisaje como el patrimonio cultural, se han convertido en una fuente de riqueza y en polo de atracción para nuevas actividades y residentes. Los motivos los hallamos en su capacidad para generar empleo e ingresos directos, pero también indirectos en sectores como el turismo, el transporte, la construcción, etc., y en su capacidad para influir en la economía y política local.

Una de las estrategias implementadas para el desarrollo local en el medio rural guarda relación con la puesta en valor del paisaje y del patrimonio, a través del turismo cultural. Lejos de pensar que este tipo de turismo concierne exclusivamente al patrimonio monumental, cualquier tipo de manifestación cultural organizada y potenciada por un mediador, 
puede convertirse en una fuente de creación ingresos, además de fomentar el conocimiento de distintas culturas, tradiciones y modos de vida.

Las galerías drenantes son un extraordinario al tiempo que singular, ejemplo de patrimonio cultural asociado a la agricultura de regadío. De hecho, en numerosos espacios constituyen el componente primigenio de los sistemas de regadío y del paisaje asociado, al ser el origen de las aguas del sistema. Se tratan de unos elementos pocos conocidos fuera del contexto agropecuario; pero más frecuentes de lo imaginado, especialmente en la cuenca mediterránea. La escasez de agua superficial consecuencia de un clima seco y estepario como el mediterráneo, desarrolló la agudeza de las sociedades humanas que habitaban estas áreas en lo referente a la captación de agua. Agua captada para el consumo propio, pero sobre todo para distribuirla por los campos de cultivo.

Una galería drenante es una obra de ingeniería hidráulica espectacular destinada a la captación de agua subterránea desde el acuífero o del subálveo de un lecho fluvial, y a su transporte hasta el exterior. Y es espectacular por diversos motivos. En primer lugar por su antigüedad, pues es fruto del conocimiento tradicional heredado convertido en técnica por expertos de distintas civilizaciones: persas, romanos, musulmanes, cristianos... En segundo lugar por la complejidad constructiva, que exigía de numerosos cálculos y estudios del terreno. En tercer lugar por las dimensiones de la obra, alcanzando algunas galerías trazados subterráneos kilométricos y alzados abovedados revestidos de piedra seca. Y en cuarto lugar por las implicaciones económicas y paisajísticas que comportan: las bocaminas de galerías drenantes alimentan con agua acequias y canales, que abastecen a los aljibes de un asentamiento o a las albercas de un área de cultivo.

Las galerías drenantes son un elemento de nuestro patrimonio hidráulico que es necesario identificar, caracterizar y evaluar con el objetivo de proponer medidas de actuación para su protección, gestión y puesta en valor. Ya hemos apuntado que se tratan de elementos poco conocidos y reconocidos socialmente, lo que no facilita su conservación. El proyecto del Ministerio de Educación y Ciencia: «Las galerías drenantes: estudio, evaluación y revalorización de un elemento singular del patrimonio del agua en la Cuenca Hidrográfica del río Júcar (Teruel, Cuenca, Albacete y Valencia)», nos ha dado la oportunidad de seguir completando el inventario de galerías de España y de incrementar el conocimiento acerca de su funcionamiento y tipologías. Pero además nos ha permitido interpretarlas, a través de la propuesta de diseño de un parque patrimonial, como parte de un sistema patrimonial susceptible de ser asimilado como un recurso ambiental, cultural y socioeconómico.

\section{BIBLIOGRAFÍA}

ANTEQUERA, M., IRANZO, E. y HERMOSILLA, J. (2011): «Los sistemas de oasis en Túnez y su relación con las galerías drenantes», en Las galerías de agua tunecinas. Las gobernaciones de Kebili, Tozeur, Gafsa y Gabès, Edita Departament de Geografia. Universitat de València. Valencia. 148 pp.

DOMÉNECH, R. (1994): El Bocairent subterráneo (Alcavors), Trabajo inédito.

GIL, E., MARTÍNEZ, R. y GÓMEZ, J.M. (2010): «La Calle del Agua, un proyecto paisajístico en Pliego-Murcia (Sureste de España), en Actas del VI Congreso Internacional de Ordenación del Territorio. Pamplona, pp. 1-14.

GOBLOT, H. (1979): Les Qanats, une technique d'acquisition de l'eau, École des Hautes Études en Sciencies Sociales, Ed. Mouton. París.

GÓMEZ, J., GIL, E., LÓPEZ, J.A., MARTÍNEZ, R. y ALIAGA, I. (2009): Paisaje y patrimonio generados por galerías y minados en la Región de Murcia. Colección Usos del Agua en el Territorio, $\mathrm{n}^{\circ}$ 5. Servicio de Publicaciones, Universidad de Murcia, $175 \mathrm{pp}$. 
HERMOSILLA, J. (Dir.) (2006): Las galerías drenantes del Sureste de la Península Ibérica. Uso tradicional del agua y sostenibilidad en el Mediterráneo español, Ed. Ministerio de Medio Ambiente, Madrid. 228 pp.

HERMOSILLA, J. E IRANZO, E. (2009): «El diseño de un parque patrimonial municipal como estrategia de desarrollo local. Los casos de Cortes de Pallás y de Arcos de las Salinas», en El desarrollo regional en periodos de cambio. Congreso de la Asociación Española de Ciencia Regional. XXXV Reunión de Estudios Regionales, Valencia, pp. $1-14$.

HERMOSILLA, J. E IRANZO, E. (2010): «Censo de hidráulica tradicional en el mediterráneo peninsular», en Áreas. Revista Internacional de Ciencias Sociales, $\mathrm{n}^{\circ}$ 29, pp. $72-89$.

IRANZO, E. (2004): «Las foggaras o minas de agua», en HERMOSILLA, J. (dir.) La Arquitectura del Agua en el riu Magre: Alcalans-Marquesat. Edita DGPCV, Conselleria de Cultura i Educació. Generalitat Valenciana. Valencia, pp. 163-178.

KOBORI, I. (1995): Water System in Arid Lands, Meiji University. Tokio.

LAUREANO, P. (2001): Atlante d'acqua, conoscenze tradizionali per la lotta alla desertificazione, Bollati Boringhieri. Torino, $424 \mathrm{pp}$.

PALENZUELA, P., COBACHO, M.A. y GUZMÁN, M. (Coord.) (2000): Guía para la puesta en valor del patrimonio del Medio Rural, Edita Junta de Andalucía. Consejería de Agricultura y Pesca. Empresa Pública para el Desarrollo Agrario y Pesquero de Andalucía.

PEREDA HERNÁNDEZ, M.J. (1987): «Pugna entre los Concejos de Chinchilla y Almansa por las Aguas de Alpera. Mediación de Don Juan Pacheco y Sentencia arbitral de 29-9-1458». Congreso de Historia del Señorío de Villena, Albacete 23-26 de octubre de 1986. Ed. Instituto de Estudios Albacetenses de la Excma. Diputación de Albacete, C.S.I.C, pp. 275-282.

PEREDA HERNÁNDEZ, M.J. (1992): «La Comunidad de Regantes de las Aguas del Pantano de Almansa».

PEREDA HERNÁNDEZ, M.J. (1996): «El Pantano de Almansa: patrimonio municipal». Revista de Fiestas de Almansa, 1996.

SABATÉ, J. (2005): «De la preservación del patrimonio a la ordenación del paisaje», en Identidades. Territorio, cultura, patrimonio, Laboratorio Internacional del Paisajes Culturales, $\mathrm{n}^{\circ} 1$, pp. 15-33.

SABATÉ, J. (2011): «De la Preservación del Patrimonio a la Ordenación del Paisaje. Intervenciones en Paisajes Culturales en Latinoamérica», en Paisajes Culturales: comprensión, protección y gestión, Edita Programa P>D Patrimonio para el Desarrollo AECID, pp. 10-23.

TARONCHER, V. (1984): «Alpera ayer y hoy. Las aguas de Alpera (I)», en Malecón, periódico mensual de información local de Alpera, Año II, No 6, febrero de 1984.

TARONCHER, V. (1984): «Alpera ayer y hoy. El pleito de las aguas (II)», en Malecón, periódico mensual de información local de Alpera, Año II, No 7 y 8, marzo-abril de 1984.

YAGO, R.; JORDÀ, R.; SOLER, A. (2004): El patrimoni arquitectònic de la Ciutat d'Albaida. Ed. Excmo. Ajuntament d'Albaida. 
